CUBO, A Mathematical Journal

Vol.22, $\mathrm{N}^{\circ} 02,(257-271)$. August 2020

Received: 07 November, 2019 | Accepted: 20 July, 2020

\title{
Results on para-Sasakian manifold admitting a quarter symmetric metric connection
}

\author{
VishnuVARDhana. S.V. ${ }^{1}$ AND VENKATEShA ${ }^{2}$ \\ ${ }^{1}$ Department of Mathematics, GITAM School of Science, GITAM (Deemed to be University) \\ Bengaluru, Karnataka-561 203, INDIA. \\ ${ }^{2}$ Department of Mathematics, Kuvempu University, Shankaraghatta - 577 451, Shimoga, \\ Karnataka, INDIA. \\ svvishnuvardhana@gmail.com, vensmath@gmail.com
}

\begin{abstract}
In this paper we have studied pseudosymmetric, Ricci-pseudosymmetric and projectively pseudosymmetric para-Sasakian manifold admitting a quarter-symmetric metric connection and constructed examples of 3-dimensional and 5-dimensional para-Sasakian manifold admitting a quarter-symmetric metric connection to verify our results.

\section{RESUMEN}

En este artículo hemos estudiado variedades para-Sasakianas seudosimétricas, Ricciseudosimétricas y proyectivamente seudosimétricas que admiten una conexión métrica cuarto-simétrica, y construimos ejemplos de variedades para-Sasakianas 3-dimensional y 5-dimensional que admiten una conexión métrica cuarto-simétrica para verificar nuestros resultados.
\end{abstract}

Keywords and Phrases: Para-Sasakian manifold, pseudosymmetric, Ricci-pseudosymmetric, projectively pseudosymmetric, quarter-symmetric metric connection.

2020 AMS Mathematics Subject Classification: 53C35, 53D40. 


\section{Introduction}

One of the most important geometric property of a space is symmetry. Spaces admitting some sense of symmetry play an important role in differential geometry and general relativity. Cartan [5] introduced locally symmetric spaces, i.e., the Riemannian manifold $(M, g)$ for which $\nabla R=0$, where $\nabla$ denotes the Levi-Civita connection of the metric. The integrability condition of $\nabla R=0$ is $R \cdot R=0$. Thus, every locally symmetric space satisfies $R \cdot R=0$, whereby the first $\mathrm{R}$ stands for the curvature operator of $(M, g)$, i.e., for tangent vector fields $X$ and $Y$ one has $R(X, Y)=$ $\nabla_{X} \nabla_{Y}-\nabla_{Y} \nabla_{X}-\nabla_{[X, Y]}$, which acts as a derivation on the second $R$ which stands for the RiemannChristoffel curvature tensor. The converse however does not hold in general. The spaces for which $R \cdot R=0$ holds at every point were called semi-symmetric spaces and which were classified by Szabo [19].

Semisymmetric manifolds form a subclass of the class of pseudosymmetric manifolds. In some spaces $R \cdot R$ is not identically zero, these turn out to be the pseudo-symmetric spaces of Deszcz [9, 10, 11, which were characterized by the condition $R \cdot R=L Q(g, R)$, where $L$ is a real function on $M$ and $Q(g, R)$ is the Tachibana tensor of $M$.

If at every point of $M$ the curvature tensor satisfies the condition

$$
R(X, Y) \cdot \mathcal{J}=L_{\mathcal{J}}\left[\left(X \wedge_{g} Y\right) \cdot \mathcal{J}\right]
$$

then a Riemannian manifold $M$ is called pseudosymmetric (resp., Ricci-pseudosymmetric, projectively pseudosymmetric) when $\mathcal{J}=R($ resp., $S, P)$. Here $\left(X \wedge_{g} Y\right)$ is an endomorphism and is defined by $\left(X \wedge_{g} Y\right) Z=g(Y, Z) X-g(X, Z) Y$ and $L_{\mathcal{J}}$ is some function on $U_{\mathcal{J}}=\{x \in M: \mathcal{J} \neq 0\}$ at $x$. A geometric interpretation of the notion of pseudosymmetry is given in [13. It is also easy to see that every pseudosymmetric manifold is Ricci-pseudosymmetric, but the converse is not true.

An analogue to the almost contact structure, the notion of almost paracontact structure was introduced by Sato [18. An almost contact manifold is always odd-dimensional but an almost paracontact manifold could be of even dimension as well. Kaneyuki and Williams [14 studied the almost paracontact structure on a pseudo-Riemannian manifold. Recently, almost paracontact geometry in particular, para-Sasakian geometry has taking interest, because of its interplay with the theory of para-Kahler manifolds and its role in pseudo-Riemannian geometry and mathematical physics ([4, 7, 8], etc.,).

As a generalization of semi-symmetric connection, quarter-symmetric connection was introduced. Quarter-symmetric connection on a differentiable manifold with affine connection was defined and studied by Golab [12. From thereafter many geometers studied this connection on different manifolds.

Para-Sasakian manifold with respect to quarter-symmetric metric connection was studied by 
De et.al., 16, 1], Pradeep Kumar et.al., [17] and Bisht and Shanker [15].

Motivated by the above studies in this article we study properties of projective curvature tensor on para-Sasakian manifold admitting a quarter-symmetric metric connection. The organization of the paper is as follows: In Section 2, we present some basic notions of para-Sasakian manifold and quarter-symmetric metric connection on it. Section 3 and 4 are respectively devoted to study the pseudosymmetric and Ricci-pseudosymmetric para-Sasakian manifold admitting a quarter-symmetric metric connection. Here we prove that if a para-Sasakian manifold $M^{n}$ admitting a quarter-symmetric metric connection is Pseudosymmetric (resp., Ricci pseudosymmetric) then $M^{n}$ is an Einstein manifold with respect to quarter-symmetric metric connection or it satisfies

$L_{\tilde{R}}=-2$ (resp., $\left.L_{\tilde{S}}=-2\right)$. Section 5 and 6 are concerned with projectively flat and projectively pseudosymmetric para-Sasakian manifold $M^{n}$ admitting a quarter-symmetric metric connection. Finally, we construct examples of 3-dimensional and 5-dimensional para-Sasakian manifold admitting a quarter-symmetric metric connection and we find some of its geometric characteristics.

\section{Preliminaries}

A differential manifold $M^{n}$ is said to admit an almost paracontact Riemannian structure $(\phi, \xi, \eta, g)$, where $\phi$ is a tensor field of type $(1,1), \xi$ is a vector field, $\eta$ is a 1 -form and $g$ is a Riemannian metric on $M^{n}$ such that

$$
\begin{array}{r}
\phi^{2} X=X-\eta(X) \xi, \quad \eta(\xi)=1, \quad \phi(\xi)=0, \quad \eta(\phi X)=0, \\
g(X, \xi)=\eta(X), \quad g(\phi X, \phi Y)=g(X, Y)-\eta(X) \eta(Y),
\end{array}
$$

for all vector fields $X, Y \in \chi\left(M^{n}\right)$. If $(\phi, \xi, \eta, g)$ on $M^{n}$ satisfies the following equations

$$
\begin{array}{r}
\left(\nabla_{X} \phi\right) Y=-g(X, Y) \xi-\eta(Y) X+2 \eta(X) \eta(Y) \xi \\
d \eta=0 \quad \text { and } \quad \nabla_{X} \xi=\phi X
\end{array}
$$

then $M^{n}$ is called para-Sasakian manifold [3].

In a para-Sasakian manifold, the following relations hold [6]:

$$
\begin{array}{r}
\left(\nabla_{X} \eta\right) Y=-g(X, Y)+\eta(X) \eta(Y), \\
\eta(R(X, Y) Z)=g(X, Z) \eta(Y)-g(Y, Z) \eta(X), \\
R(X, Y) \xi=\eta(X) Y-\eta(Y) X, \quad R(\xi, X) Y=\eta(Y) X-g(X, Y) \xi, \\
S(X, \xi)=-(n-1) \eta(X), \\
S(\phi X, \phi Y)=S(X, Y)+(n-1) \eta(X) \eta(Y),
\end{array}
$$

for every vector fields $X, Y, Z$ on $M^{n}$. Here $\nabla$ denotes the Levi-Civita connection, $R$ denotes the Riemannian curvature tensor and $\mathrm{S}$ denotes the Ricci curvature tensor. 
Here we consider a quarter-symmetric metric connection $\tilde{\nabla}$ on a para-Sasakian manifold [16] given by

$$
\tilde{\nabla}_{X} Y=\nabla_{X} Y+\eta(Y) \phi X-g(\phi X, Y) \xi
$$

The relation between curvature tensor $\tilde{R}(X, Y) Z$ of $M^{n}$ with respect to quarter-symmetric metric connection $\tilde{\nabla}$ and the curvature tensor $R(X, Y) Z$ with respect to the Levi-Civita connection $\nabla$ is given by

$$
\begin{array}{r}
\tilde{R}(X, Y) Z=R(X, Y) Z+3 g(\phi X, Z) \phi Y-3 g(\phi Y, Z) \phi X \\
+\{\eta(X) Y-\eta(Y) X\} \eta(Z)-[g(Y, Z) \eta(X)-\eta(Y) g(X, Z)] \xi .
\end{array}
$$

Also from (2.11) we obtain

$$
\tilde{S}(Y, Z)=S(Y, Z)+2 g(Y, Z)-(n+1) \eta(Y) \eta(Z)-3 \operatorname{trace} \phi g(\phi Y, Z),
$$

where $\tilde{S}$ and $S$ are Ricci tensors of connections $\tilde{\nabla}$ and $\nabla$ respectively.

\section{Pseudosymmetric para-Sasakian manifold admitting a quarter- symmetric metric connection}

A para-Sasakian manifold $M^{n}$ admitting a quarter-symmetric metric connection is said to be pseudosymmetric if

$$
\tilde{R}(X, Y) \cdot \tilde{R}=L_{\tilde{R}}\left[\left(X \wedge_{g} Y\right) \cdot \tilde{R}\right],
$$

holds on the set $U_{\tilde{R}}=\left\{x \in M^{n}: \tilde{R} \neq 0\right.$ at $\left.x\right\}$, where $L_{\tilde{R}}$ is some function on $U_{\tilde{R}}$.

Suppose that $M^{n}$ be pseudosymmetric, then in view of (3.1) we have

$$
\begin{array}{r}
\tilde{R}(\xi, Y) \tilde{R}(U, V) W-\tilde{R}(\tilde{R}(\xi, Y) U, V) W-\tilde{R}(U, \tilde{R}(\xi, Y) V) W \\
-\tilde{R}(U, V) \tilde{R}(\xi, Y) W=L_{\tilde{R}}\left[\left(\xi \wedge_{g} Y\right) \tilde{R}(U, V) W-\tilde{R}\left(\left(\xi \wedge_{g} Y\right) U, V\right) W\right. \\
\left.-\tilde{R}\left(U,\left(\xi \wedge_{g} Y\right) V\right) W-\tilde{R}(U, V)\left(\xi \wedge_{g} Y\right) W\right] .
\end{array}
$$

By virtue of (2.7) and (2.11), (3.2) takes the form

$$
\begin{gathered}
\left(L_{\tilde{R}}+2\right)[\eta(\tilde{R}(U, V) W) Y-g(Y, \tilde{R}(U, V) W) \xi-\eta(U) \tilde{R}(Y, V) W+g(Y, U) \tilde{R}(\xi, V) W \\
-\eta(V) \tilde{R}(U, Y) W+g(Y, V) \tilde{R}(U, \xi) W-\eta(W) \tilde{R}(U, V) Y+g(Y, W) \tilde{R}(U, V) \xi]=0 .
\end{gathered}
$$

Taking inner product of (3.3) with $\xi$ and using (2.6) and (2.11), we get

$$
\begin{array}{r}
\left(L_{\tilde{R}}+2\right)[g(Y, R(U, V) W)+3 g(\phi U, W) g(\phi V, Y)-3 g(\phi V, W) g(\phi U, Y) \\
+\eta(W)\{\eta(U) g(V, Y)-\eta(V) g(U, Y)\}-\{g(V, W) \eta(U)-\eta(V) g(U, W)\} \eta(Y) \\
+2\{g(V, W) g(Y, U)-g(V, Y) g(U, W)\}]=0 .
\end{array}
$$


Assuming that $L_{\tilde{R}}+2 \neq 0$, the above equation becomes

$$
\begin{array}{r}
g(Y, R(U, V) W)+3 g(\phi U, W) g(\phi V, Y)-3 g(\phi V, W) g(\phi U, Y) \\
+\eta(W)\{\eta(U) g(V, Y)-\eta(V) g(U, Y)\}-[g(V, W) \eta(U)-\eta(V) g(U, W)] \eta(Y) \\
+2[g(V, W) g(Y, U)-g((V, Y) g(U, W)]=0 .
\end{array}
$$

Putting $V=W=e_{i}$, where $\left\{e_{i}\right\}$ is an orthonormal basis of the tangent space at each point of the manifold and taking summation over $i, i=1,2,3, \cdots, n$, we get

$$
\tilde{S}(Y, U)=-2(n-1) g(Y, U) .
$$

Hence, we can state the following:

Theorem 1. If a para-Sasakian manifold $M^{n}$ admitting a quarter-symmetric metric connection is pseudosymmetric then $M^{n}$ is an Einstein manifold with respect to quarter-symmetric metric connection or it satisfies $L_{\tilde{R}}=-2$.

\section{Ricci-pseudosymmetric para-Sasakian manifold admitting a quarter-symmetric metric connection}

A para-Sasakian manifold $M^{n}$ admitting a quarter-symmetric metric connection is said to be Ricci-pseudosymmetric if the following condition is satisfied

$$
\tilde{R}(X, Y) \cdot \tilde{S}=L_{\tilde{S}}\left[\left(X \wedge_{g} Y\right) \cdot \tilde{S}\right]
$$

on $U_{\tilde{S}}$.

Let para-Sasakian manifold $M^{n}$ admitting a quarter-symmetric metric connection be Riccipseudosymmetric. Then we have

$$
\tilde{S}(\tilde{R}(X, Y) Z, W)+\tilde{S}(Z, \tilde{R}(X, Y) W)=L_{\tilde{S}}\left[\tilde{S}\left(\left(X \wedge_{g} Y\right) Z, W\right)+\tilde{S}\left(Z,\left(X \wedge_{g} Y\right) W\right)\right] .
$$

By taking $Y=W=\xi$ and making use of (2.7), (2.8) and (2.11), the above equation turns into

$$
\left(L_{\tilde{S}}+2\right)[\tilde{S}(X, Z)+2(n-1) g(X, Z)]=0
$$

Thus, we have the following assertion:

Theorem 2. If a para-Sasakian manifold $M^{n}$ admitting a quarter-symmetric metric connection is Ricci-pseudosymmetric then $M^{n}$ is an Einstein manifold with respect to quarter-symmetric metric connection or it satisfies $L_{\tilde{S}}=-2$. 


\section{Projectively flat para-Sasakian manifold admitting a quarter- symmetric metric connection}

The projective curvature tensor on a Riemannian manifold is defined by [2]

$$
P(X, Y) Z=R(X, Y) Z-\frac{1}{(n-1)}[S(Y, Z) X-S(X, Z) Y]
$$

For an $n$-dimensional para-Sasakian manifold $M^{n}$ admitting a quarter-symmetric metric connection, the projective curvature tensor is given by

$$
\tilde{P}(X, Y) Z=\tilde{R}(X, Y) Z-\frac{1}{(n-1)}[\tilde{S}(Y, Z) X-\tilde{S}(X, Z) Y] .
$$

Theorem 3. A projectively flat para-Sasakian manifold $M^{n}$ admitting a quarter-symmetric metric connection is an Einstein manifold with respect to quarter-symmetric metric connection.

Proof. Consider a projectively flat para-Sasakian manifold admitting a quarter-symmetric metric connection. Then from (5.2) we have

$$
g(\tilde{R}(X, Y) Z, W)=\frac{1}{(n-1)}[\tilde{S}(Y, Z) g(X, W)-\tilde{S}(X, Z) g(Y, W)] .
$$

Setting $X=W=\xi$ in (5.3) and using (2.7), (2.8), (2.11) and (2.12), we get

$$
\tilde{S}(X, Z)=-2(n-1) g(X, Z) .
$$

Hence, the proof is completed.

\section{Projectively pseudosymmetric para-Sasakian manifold ad- mitting a quarter-symmetric metric connection}

A para-Sasakian manifold admitting a quarter-symmetric metric connection is said to be projectively pseudosymmetric if

$$
\tilde{R}(X, Y) \cdot \tilde{P}=L_{\tilde{P}}\left[\left(X \wedge_{g} Y\right) \cdot \tilde{P}\right],
$$

holds on the set $U_{\tilde{P}}=\left\{x \in M^{n}: \tilde{P} \neq 0\right.$ at $\left.x\right\}$, where $L_{\tilde{P}}$ is some function on $U_{\tilde{P}}$.

Let $M^{n}$ be projectively pseudosymmetric, then we have

$$
\begin{array}{r}
\tilde{R}(X, \xi) \tilde{P}(U, V) \xi-\tilde{P}(\tilde{R}(X, \xi) U, V) \xi-\tilde{P}(U, \tilde{R}(X, \xi) V) \xi \\
-\tilde{P}(U, V) \tilde{R}(X, \xi) \xi=L_{\tilde{P}}\left[\left(X \wedge_{g} \xi\right) \tilde{P}(U, V) \xi-\tilde{P}\left(\left(X \wedge_{g} \xi\right) U, V\right) \xi\right. \\
\left.-\tilde{P}\left(U,\left(X \wedge_{g} \xi\right) V\right) \xi-\tilde{P}(U, V)\left(X \wedge_{g} \xi\right) \xi\right] .
\end{array}
$$


By virtue of (2.11), (2.12) and (5.2), (6.2) becomes

$$
\left(L_{\tilde{P}}+2\right) \tilde{P}(U, V) X=0 .
$$

So, one can state that:

Theorem 4. If a para-Sasakian manifold $M^{n}$ admitting a quarter-symmetric metric connection is projectively pseudosymmetric then $M^{n}$ is projectively flat with respect to quarter-symmetric metric connection or $L_{\tilde{P}}=-2$.

In view of theorem [3, one can state the above theorem as

Theorem 5. If a para-Sasakian manifold $M^{n}$ admitting a quarter-symmetric metric connection is projectively pseudosymmetric then $M^{n}$ is an Einstein manifold with respect to quarter-symmetric metric connection or $L_{\tilde{P}}=-2$.

\section{$7 \quad$ Examples}

\subsection{Example}

We consider a 3 -dimensional manifold $M=\left\{(x, y, z) \in \mathbb{R}^{3}: z \neq 0\right\}$, where $(x, y, z)$ are standard coordinates in $\mathbb{R}^{3}$. Let $\left\{E_{1}, E_{2}, E_{3}\right\}$ be a linearly independent global frame field on $M$ given by

$$
E_{1}=e^{z} \frac{\partial}{\partial y}, \quad E_{2}=e^{z}\left(\frac{\partial}{\partial y}-\frac{\partial}{\partial x}\right), \quad E_{3}=\frac{\partial}{\partial z},
$$

If $g$ is a Riemannian metric defined by

$$
g\left(E_{i}, E_{j}\right)= \begin{cases}1, & i=j \\ 0, & i \neq j\end{cases}
$$

for $1 \leq i, j \leq 3$, and if $\eta$ is the 1 -form defined by $\eta(Z)=g\left(Z, E_{3}\right)$ for any vector field $Z \in \chi(M)$. We define the (1, 1)-tensor field $\phi$ as

$$
\phi\left(E_{1}\right)=E_{1}, \quad \phi\left(E_{2}\right)=-E_{2}, \quad \phi\left(E_{3}\right)=0 .
$$

The linearity property of $\phi$ and $g$ yields that

$$
\begin{aligned}
& \eta\left(E_{3}\right)=1, \\
& \phi^{2} U=U-\eta(U) E_{3}, \\
& g(\phi U, \phi V)=g(U, V)-\eta(U) \eta(V),
\end{aligned}
$$


for any $U, V \in \chi(M)$.

Now we have

$$
\left[E_{1}, E_{2}\right]=0, \quad\left[E_{1}, E_{3}\right]=E_{1}, \quad\left[E_{2}, E_{3}\right]=E_{2}
$$

The Riemannian connection $\nabla$ of the metric $g$ known as Koszul's formula and is given by

$$
\begin{array}{r}
2 g\left(\nabla_{X} Y, Z\right)=X g(Y, Z)+Y g(Z, X)-Z g(X, Y)-g(X,[Y, Z]) \\
-g(Y,[X, Z])+g(Z,[X, Y]) .
\end{array}
$$

Using Koszul's formula we get the followings in matrix form

$$
\left(\begin{array}{ccc}
\nabla_{E_{1}} E_{1} & \nabla_{E_{1}} E_{2} & \nabla_{E_{1}} E_{3} \\
\nabla_{E_{2}} E_{1} & \nabla_{E_{2}} E_{2} & \nabla_{E_{2}} E_{3} \\
\nabla_{E_{3}} E_{1} & \nabla_{E_{3}} E_{2} & \nabla_{E_{3}} E_{3}
\end{array}\right)=\left(\begin{array}{ccc}
-E_{3} & 0 & E_{1} \\
0 & -E_{3} & E_{2} \\
0 & 0 & 0
\end{array}\right) .
$$

Clearly $(\phi, \xi, \eta, g)$ is a para-Sasakian structure on $M$. Thus $M(\phi, \xi, \eta, g)$ is a 3 -dimensional para-Sasakian manifold.

Using (2.10) and the above equation, one can easily obtain the following:

$$
\left(\begin{array}{ccc}
\tilde{\nabla}_{E_{1}} E_{1} & \tilde{\nabla}_{E_{1}} E_{2} & \tilde{\nabla}_{E_{1}} E_{3} \\
\tilde{\nabla}_{E_{2}} E_{1} & \tilde{\nabla}_{E_{2}} E_{2} & \tilde{\nabla}_{E_{2}} E_{3} \\
\tilde{\nabla}_{E_{3}} E_{1} & \tilde{\nabla}_{E_{3}} E_{2} & \tilde{\nabla}_{E_{3}} E_{3}
\end{array}\right)=\left(\begin{array}{ccc}
-2 E_{3} & 0 & 2 E_{1} \\
0 & -2 E_{3} & 2 E_{2} \\
0 & 0 & 0
\end{array}\right) .
$$

With the help of the above results it can be easily verified that

$$
\begin{array}{lll}
R\left(E_{1}, E_{2}\right) E_{3}=0, & R\left(E_{2}, E_{3}\right) E_{3}=-E_{2}, & R\left(E_{1}, E_{3}\right) E_{3}=-E_{1}, \\
R\left(E_{1}, E_{2}\right) E_{2}=-E_{1}, & R\left(E_{2}, E_{3}\right) E_{2}=E_{3}, & R\left(E_{1}, E_{3}\right) E_{2}=0, \\
R\left(E_{1}, E_{2}\right) E_{1}=E_{2}, & R\left(E_{2}, E_{3}\right) E_{1}=0, & R\left(E_{1}, E_{3}\right) E_{1}=E_{3} .
\end{array}
$$

and

$$
\begin{aligned}
& \tilde{R}\left(E_{1}, E_{2}\right) E_{3}=0, \quad \tilde{R}\left(E_{2}, E_{3}\right) E_{3}=-2 E_{2}, \quad \tilde{R}\left(E_{1}, E_{3}\right) E_{3}=-2 E_{1}, \\
& \tilde{R}\left(E_{1}, E_{2}\right) E_{2}=-4 E_{1}, \quad \tilde{R}\left(E_{2}, E_{3}\right) E_{2}=2 E_{3}, \quad \tilde{R}\left(E_{1}, E_{3}\right) E_{2}=0, \\
& \tilde{R}\left(E_{1}, E_{2}\right) E_{1}=4 E_{2}, \quad \tilde{R}\left(E_{2}, E_{3}\right) E_{1}=0, \quad \tilde{R}\left(E_{1}, E_{3}\right) E_{1}=2 E_{3} .
\end{aligned}
$$

Since $E_{1}, E_{2}, E_{3}$ forms a basis, any vector field $X, Y, Z \in \chi(M)$ can be written as $X=$ $a_{1} E_{1}+b_{1} E_{2}+c_{1} E_{3}, Y=a_{2} E_{1}+b_{2} E_{2}+c_{2} E_{3}, Z=a_{3} E_{1}+b_{3} E_{2}+c_{3} E_{3}$, where $a_{i}, b_{i}, c_{i} \in \mathbb{R}$, $i=1,2,3$. Using the expressions of the curvature tensors, we find values of Riemannian curvature 
and Ricci curvature with respect to quarter-symmetric metric connection as;

$$
\begin{aligned}
\tilde{R}(X, Y) Z & =\left[-4\left\{a_{1} b_{2}-b_{1} a_{2}\right\} b_{3}+2\left\{c_{1} a_{2}-a_{1} c_{2}\right\} c_{3}\right] E_{1} \\
& +\left[-4\left\{b_{1} a_{2}-a_{1} b_{2}\right\} a_{3}+2\left\{c_{1} b_{2}-b_{1} c_{2}\right\} c_{3}\right] E_{2} \\
& +\left[-2\left\{c_{1} a_{2}-a_{1} c_{2}\right\} a_{3}-2\left\{c_{1} b_{2}-b_{1} c_{2}\right\} b_{3}\right] E_{3} \\
\tilde{S}\left(E_{1}, E_{1}\right) & =\tilde{S}\left(E_{2}, E_{2}\right)=-6, \tilde{S}\left(E_{3}, E_{3}\right)=-4 .
\end{aligned}
$$

Using (7.1), (7.3) and the expression of the endomorphism $\left(X \wedge_{g} Y\right) Z=g(Y, Z) X-g(X, Z) Y$, one can easily verify that

$$
\tilde{S}\left(\tilde{R}\left(X, E_{3}\right) Y, E_{3}\right)+\tilde{S}\left(Y, \tilde{R}\left(X, E_{3}\right) E_{3}\right)=-2\left[\tilde{S}\left(\left(X \wedge_{g} E_{3}\right) Y, E_{3}\right)+\tilde{S}\left(Y,\left(X \wedge_{g} E_{3}\right) E_{3}\right)\right],
$$

here $L_{\tilde{S}}=-2$. Thus, the above equation verify one part of the Theorem 2 of section 4 ,

Moreover, the manifold under consideration satisfies

$$
\begin{array}{r}
\tilde{R}(X, Y) Z=-\tilde{R}(Y, X) Z, \\
\tilde{R}(X, Y) Z+\tilde{R}(Y, Z) X+\tilde{R}(Z, X) Y=0 .
\end{array}
$$

Hence, from the above equations one can say that this example verifies the condition $(c)$ of Theorem 3.1 in [1] and first Bianchi identity.

\subsection{Example}

We consider a 5-dimensional manifold $M=\left\{\left(x_{1}, x_{2}, x_{3}, x_{4}, x_{5}\right) \in \mathbb{R}^{5}\right\}$, where $\left(x_{1}, x_{2}, x_{3}, x_{4}, x_{5}\right)$ are standard coordinates in $\mathbb{R}^{5}$. We choose the vector fields

$$
E_{1}=\frac{\partial}{\partial x_{1}}, \quad E_{2}=\frac{\partial}{\partial x_{2}}, \quad E_{3}=\frac{\partial}{\partial x_{3}}, \quad E_{4}=\frac{\partial}{\partial x_{4}}, \quad E_{5}=x_{1} \frac{\partial}{\partial x_{1}}+x_{2} \frac{\partial}{\partial x_{2}}+x_{3} \frac{\partial}{\partial x_{3}}+x_{4} \frac{\partial}{\partial x_{4}}+\frac{\partial}{\partial x_{5}},
$$

which are linearly independent at each point of $M$.

Let $g$ be a Riemannian metric defined by

$$
g\left(E_{i}, E_{j}\right)= \begin{cases}1, & i=j \\ 0, & i \neq j\end{cases}
$$

for $1 \leq i, j \leq 5$, and if $\eta$ is the 1 -form defined by $\eta(Z)=g\left(Z, E_{5}\right)$ for any vector field $Z \in \chi(M)$. Let $\phi$ be the $(1,1)$-tensor field defined by

$$
\phi\left(E_{1}\right)=E_{1}, \quad \phi\left(E_{2}\right)=E_{2}, \quad \phi\left(E_{3}\right)=E_{3}, \quad \phi\left(E_{4}\right)=E_{4}, \quad \phi\left(E_{5}\right)=0 .
$$


The linearity property of $\phi$ and $g$ yields that

$$
\begin{aligned}
& \eta\left(E_{5}\right)=1 \\
& \phi^{2} U=U-\eta(U) E_{5}, \\
& g(\phi U, \phi V)=g(U, V)-\eta(U) \eta(V),
\end{aligned}
$$

for any $U, V \in \chi(M)$.

Now we have

$$
\begin{array}{ll}
{\left[E_{1}, E_{2}\right]=0,} & {\left[E_{1}, E_{3}\right]=0, \quad\left[E_{1}, E_{4}\right]=0, \quad\left[E_{1}, E_{5}\right]=E_{1},} \\
{\left[E_{2}, E_{3}\right]=0,} & {\left[E_{2}, E_{4}\right]=0, \quad\left[E_{2}, E_{5}\right]=E_{2},} \\
{\left[E_{3}, E_{4}\right]=0,} & {\left[E_{3}, E_{5}\right]=E 3, \quad\left[E_{4}, E_{5}\right]=E_{4} .}
\end{array}
$$

By virtue of Koszul's formula we get the followings in matrix form

$$
\left(\begin{array}{ccccc}
\nabla_{E_{1}} E_{1} & \nabla_{E_{1}} E_{2} & \nabla_{E_{1}} E_{3} & \nabla_{E_{1}} E_{4} & \nabla_{E_{1}} E_{5} \\
\nabla_{E_{2}} E_{1} & \nabla_{E_{2}} E_{2} & \nabla_{E_{2}} E_{3} & \nabla_{E_{2}} E_{4} & \nabla_{E_{2}} E_{5} \\
\nabla_{E_{3}} E_{1} & \nabla_{E_{3}} E_{2} & \nabla_{E_{3}} E_{3} & \nabla_{E_{3}} E_{4} & \nabla_{E_{3}} E_{5} \\
\nabla_{E_{4}} E_{1} & \nabla_{E_{4}} E_{2} & \nabla_{E_{4}} E_{3} & \nabla_{E_{4}} E_{4} & \nabla_{E_{4}} E_{5} \\
\nabla_{E_{5}} E_{1} & \nabla_{E_{5}} E_{2} & \nabla_{E_{5}} E_{3} & \nabla_{E_{5}} E_{4} & \nabla_{E_{5}} E_{5}
\end{array}\right)=\left(\begin{array}{ccccc}
-E_{5} & 0 & 0 & 0 & E_{1} \\
0 & -E_{5} & 0 & 0 & E_{2} \\
0 & 0 & -E_{5} & 0 & E_{3} \\
0 & 0 & 0 & -E_{5} & E_{4} \\
0 & 0 & 0 & 0 & 0
\end{array}\right) .
$$

Above expressions satisfies all the properties of para-Sasakian manifold. Thus $M(\phi, \xi, \eta, g)$ is a 5-dimensional para-Sasakian manifold.

From the above expressions and the relation of quarter symmetric metric connection and Riemannian connection, one can easily obtain the following:

$$
\left(\begin{array}{ccccc}
\tilde{\nabla}_{E_{1}} E_{1} & \tilde{\nabla}_{E_{1}} E_{2} & \tilde{\nabla}_{E_{1}} E_{3} & \tilde{\nabla}_{E_{1}} E_{4} & \tilde{\nabla}_{E_{1}} E_{5} \\
\tilde{\nabla}_{E_{2}} E_{1} & \tilde{\nabla}_{E_{2}} E_{2} & \tilde{\nabla}_{E_{2}} E_{3} & \tilde{\nabla}_{E_{2}} E_{4} & \tilde{\nabla}_{E_{2}} E_{5} \\
\tilde{\nabla}_{E_{3}} E_{1} & \tilde{\nabla}_{E_{3}} E_{2} & \tilde{\nabla}_{E_{3}} E_{3} & \tilde{\nabla}_{E_{3}} E_{4} & \tilde{\nabla}_{E_{3}} E_{5} \\
\tilde{\nabla}_{E_{4}} E_{1} & \tilde{\nabla}_{E_{4}} E_{2} & \tilde{\nabla}_{E_{4}} E_{3} & \tilde{\nabla}_{E_{4}} E_{4} & \tilde{\nabla}_{E_{4}} E_{5} \\
\tilde{\nabla}_{E_{5}} E_{1} & \tilde{\nabla}_{E_{5}} E_{2} & \tilde{\nabla}_{E_{5}} E_{3} & \tilde{\nabla}_{E_{5}} E_{4} & \tilde{\nabla}_{E_{5}} E_{5}
\end{array}\right)=\left(\begin{array}{ccccc}
-2 E_{5} & 0 & 0 & 0 & 2 E_{1} \\
0 & -2 E_{5} & 0 & 0 & 2 E_{2} \\
0 & 0 & -2 E_{5} & 0 & 2 E_{3} \\
0 & 0 & 0 & -2 E_{5} & 2 E_{4} \\
0 & 0 & 0 & 0 & 0
\end{array}\right) .
$$

With the help of the above results it can be easily obtain the non-zero components of curvature tensors as

$$
\begin{array}{llll}
R\left(E_{1}, E_{2}\right) E_{1}=E_{2}, & R\left(E_{1}, E_{2}\right) E_{2}=-E_{1}, & R\left(E_{1}, E_{3}\right) E_{1}=E_{3}, & R\left(E_{1}, E_{3}\right) E_{3}=-E_{1}, \\
R\left(E_{1}, E_{4}\right) E_{1}=E_{4}, & R\left(E_{1}, E_{4}\right) E_{4}=-E_{1}, & R\left(E_{1}, E_{5}\right) E_{1}=E_{5}, & R\left(E_{1}, E_{5}\right) E_{5}=-E_{1}, \\
R\left(E_{2}, E_{3}\right) E_{2}=E_{3}, & R\left(E_{2}, E_{3}\right) E_{3}=-E_{2}, & R\left(E_{2}, E_{4}\right) E_{2}=E_{4}, & R\left(E_{2}, E_{4}\right) E_{4}=-E_{2}, \\
R\left(E_{2}, E_{5}\right) E_{2}=E_{5}, & R\left(E_{2}, E_{5}\right) E_{5}=-E_{2}, & R\left(E_{3}, E_{4}\right) E_{3}=E_{4}, & R\left(E_{3}, E_{4}\right) E_{4}=-E_{3}, \\
R\left(E_{3}, E_{5}\right) E_{3}=E_{5}, & R\left(E_{3}, E_{5}\right) E_{5}=-E_{3}, & R\left(E_{4}, E_{5}\right) E_{4}=E_{5}, & R\left(E_{4}, E_{5}\right) E_{5}=-E_{4},
\end{array}
$$


and

$$
\begin{array}{llll}
\tilde{R}\left(E_{1}, E_{2}\right) E_{1}=4 E_{2}, & \tilde{R}\left(E_{1}, E_{2}\right) E_{2}=-4 E_{1}, & \tilde{R}\left(E_{1}, E_{3}\right) E_{1}=4 E_{3}, & \tilde{R}\left(E_{1}, E_{3}\right) E_{3}=-4 E_{1}, \\
\tilde{R}\left(E_{1}, E_{4}\right) E_{1}=4 E_{4}, & \tilde{R}\left(E_{1}, E_{4}\right) E_{4}=-4 E_{1}, \quad \tilde{R}\left(E_{1}, E_{5}\right) E_{1}=2 E_{5}, \quad \tilde{R}\left(E_{1}, E_{5}\right) E_{5}=-2 E_{1}, \\
\tilde{R}\left(E_{2}, E_{3}\right) E_{2}=4 E_{3}, \quad \tilde{R}\left(E_{2}, E_{3}\right) E_{3}=-4 E_{2}, \quad \tilde{R}\left(E_{2}, E_{4}\right) E_{2}=4 E_{4}, \quad \tilde{R}\left(E_{2}, E_{4}\right) E_{4}=-4 E_{2}, \\
\tilde{R}\left(E_{2}, E_{5}\right) E_{2}=2 E_{5}, \quad \tilde{R}\left(E_{2}, E_{5}\right) E_{5}=-2 E_{2}, \quad \tilde{R}\left(E_{3}, E_{4}\right) E_{3}=4 E_{4}, \quad \tilde{R}\left(E_{3}, E_{4}\right) E_{4}=-4 E_{3}, \\
\tilde{R}\left(E_{3}, E_{5}\right) E_{3}=2 E_{5}, \tilde{R}\left(E_{3}, E_{5}\right) E_{5}=-2 E_{3}, \tilde{R}\left(E_{4}, E_{5}\right) E_{4}=2 E_{5}, \tilde{R}\left(E_{4}, E_{5}\right) E_{5}=-2 E_{4} . \quad(7.5)
\end{array}
$$

Since $E_{1}, E_{2}, E_{3}, E_{4}, E_{5}$ forms a basis, any vector field $X, Y, Z \in \chi(M)$ can be written as $X=a_{1} E_{1}+b_{1} E_{2}+c_{1} E_{3}+d_{1} E_{4}+f_{1} E_{5}, Y=a_{2} E_{1}+b_{2} E_{2}+c_{2} E_{3}+d_{2} E_{4}+f_{2} E_{5}, Z=a_{3} E_{1}+$ $b_{3} E_{2}+c_{3} E_{3}+d_{3} E_{4}+f_{3} E_{5}$, where $a_{i}, b_{i}, c_{i}, d_{i}, f_{i} \in \mathbb{R}, i=1,2,3,4,5$. Using the expressions of the curvature tensors, we find values of Riemannian curvature and Ricci curvature with respect to quarter-symmetric metric connection as;

$$
\begin{aligned}
\tilde{R}(X, Y) Z & =\left[-4\left\{a_{1}\left(b_{2} b_{3}+c_{2} c_{3}+d_{2} d_{3}\right)-a_{2}\left(b_{1} b_{3}+c_{1} c_{3}+d_{1} d_{3}\right)\right\}-2\left(a_{1} f_{2}-f_{1} a_{2}\right) f_{3}\right] E_{1} \\
& +\left[-4\left\{b_{1}\left(a_{2} a_{3}+c_{2} c_{3}+d_{2} d_{3}\right)-b_{2}\left(a_{1} a_{3}+c_{1} c_{3}+d_{1} d_{3}\right)\right\}-2\left(b_{1} f_{2}-f_{1} b_{2}\right) f_{3}\right] E_{2} \\
& +\left[-4\left\{c_{1}\left(a_{2} a_{3}+b_{2} b_{3}+d_{2} d_{3}\right)-c_{2}\left(a_{1} a_{3}+b_{1} b_{3}+d_{1} d_{3}\right)\right\}-2\left(c_{1} f_{2}-f_{1} c_{2}\right) f_{3}\right] E_{3} \\
& +\left[-4\left\{d_{1}\left(a_{2} a_{3}+b_{2} b_{3}+c_{2} c_{3}\right)-d_{2}\left(a_{1} a_{3}+b_{1} b_{3}+c_{1} c_{3}\right)\right\}-2\left(d_{1} f_{2}-f_{1} d_{2}\right) f_{3}\right] E_{4} \\
& +\left[2\left\{\left(a_{1} f_{2}-f_{1} a_{2}\right) a_{3}+\left(b_{1} f_{2}-f_{1} b_{2}\right) b_{3}+\left(c_{1} f_{2}-f_{1} c_{2}\right) c_{3}+\left(d_{1} f_{2}-f_{1} d_{2}\right) d_{3}\right\}\right] E_{5}, \\
\tilde{S}\left(E_{1}, E_{1}\right) & =\tilde{S}\left(E_{2}, E_{2}\right)=\tilde{S}\left(E_{3}, E_{3}\right)=\tilde{S}\left(E_{4}, E_{4}\right)=-14, \tilde{S}\left(E_{5}, E_{5}\right)=-8 .
\end{aligned}
$$

In view of (7.5), (7.6) and the expression of the endomorphism one can easily verify the equation (7.4) and hence the Theorem 2 of section 4 is verified. This example also verifies the condition (c) of Theorem 3.1 in [1] and first Bianchi identity.

Above two examples verifies the one part of the Theorem 2, that is, if a para-Sasakian manifold $M^{n}$ admitting a quarter-symmetric metric connection is Ricci pseudosymmetric then $M^{n}$ satisfies $L_{\tilde{S}}=-2\left(M^{n}\right.$ is not Einstein manifold with respect to quarter-symmetric metric connection). Another part of the theorem is that, if a para-Sasakian manifold $M^{n}$ admitting a quarter-symmetric metric connection is Ricci pseudosymmetric then $M^{n}$ is an Einstein manifold with respect to quarter-symmetric metric connection $\left(L_{\tilde{S}} \neq-2\right)$. Now, the second part of the Theorem 2 can be verified by using the proper example.

\subsection{Example}

We consider a 5 -dimensional manifold $M=\left\{(x, y, z, u, v) \in \mathbb{R}^{5}\right\}$, where $(x, y, z, u, v)$ are standard coordinates in $\mathbb{R}^{5}$. Let $\left\{E_{1}, E_{2}, E_{3}, E_{4}, E_{5}\right\}$ be a linearly independent global frame field on $M$ given 
by

$$
E_{1}=\frac{\partial}{\partial x}, \quad E_{2}=e^{-x} \frac{\partial}{\partial y}, \quad E_{3}=e^{-x} \frac{\partial}{\partial z}, \quad E_{4}=e^{-x} \frac{\partial}{\partial u}, \quad E_{5}=e^{-x} \frac{\partial}{\partial v} .
$$

Let $g$ be a Riemannian metric defined by

$$
g\left(E_{i}, E_{j}\right)= \begin{cases}1, & i=j \\ 0, & i \neq j\end{cases}
$$

for $1 \leq i, j \leq 5$, and if $\eta$ is the 1 -form defined by $\eta(Z)=g\left(Z, E_{1}\right)$ for any vector field $Z \in \chi(M)$. Let the $(1,1)$-tensor field $\phi$ be defined by

$$
\phi\left(E_{1}\right)=0, \quad \phi\left(E_{2}\right)=E_{2}, \quad \phi\left(E_{3}\right)=E_{3}, \quad \phi\left(E_{4}\right)=E_{4}, \quad \phi\left(E_{5}\right)=E_{5} .
$$

With the help of linearity property of $\phi$ and $g$, we have

$$
\begin{aligned}
& \eta\left(E_{1}\right)=1, \\
& \phi^{2} V=V-\eta(V) E_{1}, \\
& g(\phi X, \phi Y)=g(X, Y)-\eta(X) \eta(Y),
\end{aligned}
$$

for any $X, Y \in \chi(M)$.

Now we have

$$
\begin{aligned}
& {\left[E_{1}, E_{2}\right]=-E_{2}, \quad\left[E_{1}, E_{3}\right]=-E_{3}, \quad\left[E_{1}, E_{4}\right]=-E_{4}, \quad\left[E_{1}, E_{5}\right]=-E_{5},} \\
& \left.\left[E_{2}, E_{3}\right]=\left[E_{2}, E_{4}\right]=\left[E_{2}, E_{5}\right]=\left[E_{3}, E_{4}\right]=\left[E_{3}, E_{5}\right]=E_{4}, E_{5}\right]=0 .
\end{aligned}
$$

With the help of Koszul's formula we get the followings in matrix form

$$
\left(\begin{array}{ccccc}
\nabla_{E_{1}} E_{1} & \nabla_{E_{1}} E_{2} & \nabla_{E_{1}} E_{3} & \nabla_{E_{1}} E_{4} & \nabla_{E_{1}} E_{5} \\
\nabla_{E_{2}} E_{1} & \nabla_{E_{2}} E_{2} & \nabla_{E_{2}} E_{3} & \nabla_{E_{2}} E_{4} & \nabla_{E_{2}} E_{5} \\
\nabla_{E_{3}} E_{1} & \nabla_{E_{3}} E_{2} & \nabla_{E_{3}} E_{3} & \nabla_{E_{3}} E_{4} & \nabla_{E_{3}} E_{5} \\
\nabla_{E_{4}} E_{1} & \nabla_{E_{4}} E_{2} & \nabla_{E_{4}} E_{3} & \nabla_{E_{4}} E_{4} & \nabla_{E_{4}} E_{5} \\
\nabla_{E_{5}} E_{1} & \nabla_{E_{5}} E_{2} & \nabla_{E_{5}} E_{3} & \nabla_{E_{5}} E_{4} & \nabla_{E_{5}} E_{5}
\end{array}\right)=\left(\begin{array}{ccccc}
0 & 0 & 0 & 0 & 0 \\
E_{2} & -E_{1} & 0 & 0 & 0 \\
E_{3} & 0 & -E_{1} & 0 & 0 \\
E_{4} & 0 & 0 & -E_{1} & 0 \\
E_{5} & 0 & 0 & 0 & -E_{1}
\end{array}\right) .
$$

In this case, $(\phi, \xi, \eta, g)$ is a para-Sasakian structure on $M$ and hence $M(\phi, \xi, \eta, g)$ is a 5dimensional para-Sasakian manifold.

Using (2.10) and the above equation, one can easily obtain the following:

$$
\left(\begin{array}{ccccc}
\tilde{\nabla}_{E_{1}} E_{1} & \tilde{\nabla}_{E_{1}} E_{2} & \tilde{\nabla}_{E_{1}} E_{3} & \tilde{\nabla}_{E_{1}} E_{4} & \tilde{\nabla}_{E_{1}} E_{5} \\
\tilde{\nabla}_{E_{2}} E_{1} & \tilde{\nabla}_{E_{2}} E_{2} & \tilde{\nabla}_{E_{2}} E_{3} & \tilde{\nabla}_{E_{2}} E_{4} & \tilde{\nabla}_{E_{2}} E_{5} \\
\tilde{\nabla}_{E_{3}} E_{1} & \tilde{\nabla}_{E_{3}} E_{2} & \tilde{\nabla}_{E_{3}} E_{3} & \tilde{\nabla}_{E_{3}} E_{4} & \tilde{\nabla}_{E_{3}} E_{5} \\
\tilde{\nabla}_{E_{4}} E_{1} & \tilde{\nabla}_{E_{4}} E_{2} & \tilde{\nabla}_{E_{4}} E_{3} & \tilde{\nabla}_{E_{4}} E_{4} & \tilde{\nabla}_{E_{4}} E_{5} \\
\tilde{\nabla}_{E_{5}} E_{1} & \tilde{\nabla}_{E_{5}} E_{2} & \tilde{\nabla}_{E_{5}} E_{3} & \tilde{\nabla}_{E_{5}} E_{4} & \tilde{\nabla}_{E_{5}} E_{5}
\end{array}\right)=\left(\begin{array}{ccccc}
0 & 0 & 0 & 0 & 0 \\
2 E_{2} & -2 E_{1} & 0 & 0 & 0 \\
2 E_{3} & 0 & -2 E_{1} & 0 & 0 \\
2 E_{4} & 0 & 0 & -2 E_{1} & 0 \\
2 E_{5} & 0 & 0 & 0 & -2 E_{1}
\end{array}\right) .
$$


From above results it can be easily obtain the non-zero components of Riemannian curvature and Ricci curvature tensors as

$$
\begin{array}{llll}
R\left(E_{1}, E_{2}\right) E_{1}=E_{2}, & R\left(E_{1}, E_{2}\right) E_{2}=-E_{1}, & R\left(E_{1}, E_{3}\right) E_{1}=E_{3}, & R\left(E_{1}, E_{3}\right) E_{3}=-E_{1}, \\
R\left(E_{1}, E_{4}\right) E_{1}=E_{4}, & R\left(E_{1}, E_{4}\right) E_{4}=-E_{1}, & R\left(E_{1}, E_{5}\right) E_{1}=E_{5}, & R\left(E_{1}, E_{5}\right) E_{5}=-E_{1}, \\
R\left(E_{2}, E_{3}\right) E_{2}=E_{3}, & R\left(E_{2}, E_{3}\right) E_{3}=-E_{2}, & R\left(E_{2}, E_{4}\right) E_{2}=E_{4}, & R\left(E_{2}, E_{4}\right) E_{4}=-E_{2}, \\
R\left(E_{2}, E_{5}\right) E_{2}=E_{5}, & R\left(E_{2}, E_{5}\right) E_{5}=-E_{2}, & R\left(E_{3}, E_{4}\right) E_{3}=E_{4}, & R\left(E_{3}, E_{4}\right) E_{4}=-E_{3}, \\
R\left(E_{3}, E_{5}\right) E_{3}=E_{5}, & R\left(E_{3}, E_{5}\right) E_{5}=-E_{3}, & R\left(E_{4}, E_{5}\right) E_{4}=E_{5}, & R\left(E_{4}, E_{5}\right) E_{5}=-E_{4},
\end{array}
$$

and

$$
\begin{aligned}
& \tilde{R}\left(E_{1}, E_{2}\right) E_{1}=2 E_{2}, \quad \tilde{R}\left(E_{1}, E_{2}\right) E_{2}=-2 E_{1}, \quad \tilde{R}\left(E_{1}, E_{3}\right) E_{1}=2 E_{3}, \quad \tilde{R}\left(E_{1}, E_{3}\right) E_{3}=-2 E_{1}, \\
& \tilde{R}\left(E_{1}, E_{4}\right) E_{1}=2 E_{4}, \quad \tilde{R}\left(E_{1}, E_{4}\right) E_{4}=-2 E_{1}, \quad \tilde{R}\left(E_{1}, E_{5}\right) E_{1}=2 E_{5}, \quad \tilde{R}\left(E_{1}, E_{5}\right) E_{5}=-2 E_{1}, \\
& \tilde{R}\left(E_{2}, E_{3}\right) E_{2}=2 E_{3}, \quad \tilde{R}\left(E_{2}, E_{3}\right) E_{3}=-2 E_{2}, \quad \tilde{R}\left(E_{2}, E_{4}\right) E_{2}=2 E_{4}, \quad \tilde{R}\left(E_{2}, E_{4}\right) E_{4}=-2 E_{2}, \\
& \tilde{R}\left(E_{2}, E_{5}\right) E_{2}=2 E_{5}, \quad \tilde{R}\left(E_{2}, E_{5}\right) E_{5}=-2 E_{2}, \quad \tilde{R}\left(E_{3}, E_{4}\right) E_{3}=2 E_{4}, \quad \tilde{R}\left(E_{3}, E_{4}\right) E_{4}=-2 E_{3}, \\
& \tilde{R}\left(E_{3}, E_{5}\right) E_{3}=2 E_{5}, \tilde{R}\left(E_{3}, E_{5}\right) E_{5}=-2 E_{3}, \tilde{R}\left(E_{4}, E_{5}\right) E_{4}=2 E_{5}, \tilde{R}\left(E_{4}, E_{5}\right) E_{5}=-2 E_{4}, \quad(7.7) \\
& \tilde{S}\left(E_{1}, E_{1}\right)=\tilde{S}\left(E_{2}, E_{2}\right)=\tilde{S}\left(E_{3}, E_{3}\right)=\tilde{S}\left(E_{4}, E_{4}\right)=\tilde{S}\left(E_{5}, E_{5}\right)=-8, \\
& \tilde{S}(X, Y)=-2(5-1) g(X, Y)=-8 g(X, Y),
\end{aligned}
$$

where $X=a_{1} E_{1}+b_{1} E_{2}+c_{1} E_{3}+d_{1} E_{4}+f_{1} E_{5}$ and $Y=a_{2} E_{1}+b_{2} E_{2}+c_{2} E_{3}+d_{2} E_{4}+f_{2} E_{5}$.

From (7.7), (7.8) and the expression of the endomorphism one can easily substantiate, the equation (7.4) and hence second part of the Theorem 2 (for $L_{\tilde{S}} \neq-2$ ). 


\section{References}

[1] Abul Kalam Mondal and U.C. De, Quarter-symmetric nonmetric Connection on P-Sasakian manifolds, ISRN Geometry, (2012), 1-14.

[2] G. Soos, Über die geodätischen Abbildungen von Riemannaschen Räumen auf projektivsymmetrische Riemannsche Räume, Acta. Math. Acad. Sci. Hungar., 9, (1958), 359-361

[3] A. Barman, Concircular curvature tensor on a P-Sasakian manifold admitting a quartersymmetric metric connection, Kragujevac J. Math. 42 (2018), 2, 275-285.

[4] D. V. Alekseevsky et al., Cones over pseudo-Riemannian manifolds and their holonomy, J. Reine Angew. Math. 635 (2009), 23-69.

[5] E. Cartan, Surune classe remarquable d'espaces de Riema, Soc. Math., France, 54 (1926), $214-264$.

[6] Cihan Ozgur, On A class of para-Sakakian manifolds, Turk J Math., 29 (2005), 249-257.

[7] V. Cortés et al., Special geometry of Euclidean supersymmetry. I. Vector multiplets, J. High Energy Phys., 03, (2004), 1-64.

[8] V. Cortés, M.-A. Lawn and L. Schäfer, Affine hyperspheres associated to special para-Kähler manifolds, Int. J. Geom. Methods Mod. Phys. 3 (2006), 5-6, 995-1009.

[9] R. Deszcz, On pseudosymmetric spaces, Acta Math., Hungarica, 53 (1992), 185-190.

[10] R. Deszcz and S. Yaprak, Curvature properties of certain pseudosymmetric manifolds, Publ. Math. Debrecen 45 (1994), 3-4, 333-345.

[11] R. Deszcz et al., On some curvature conditions of pseudosymmetry type, Period. Math. Hungar. 70 (2015), 2, 153-170.

[12] S. Golab, On semi-symmetric and quarter-symmetric linear connections, Tensor (N.S.) 29 (1975), 3, 249-254.

[13] S. Haesen and L. Verstraelen, Properties of a scalar curvature invariant depending on two planes, Manuscripta Math. 122 (2007), 1, 59-72.

[14] S. Kaneyuki and F. L. Williams, Almost paracontact and parahodge structures on manifolds, Nagoya Math. J. 99 (1985), 173-187.

[15] Lata Bisht and Sandhana Shanker, Curvature tensor on para-Sasakian manifold admitting quarter symmetric metric connection, IOSR Journal of Mathematics, 11(5), (2015), 22-28. 
[16] K. Mandal and U. C. De, Quarter-symmetric metric connection in a P-Sasakian manifold, An. Univ. Vest Timiş. Ser. Mat.-Inform. 53 (2015), 1, 137-150.

[17] K.T. Pradeep Kumar, Venkatesha and C.S. Bagewadi, On $\phi$-recurrent para-Sasakian manifold admitting quarter-symmetric metric connection, ISRN Geometry, (2012), 1-10.

[18] I. Sato, On a structure similar to the almost contact structure, Tensor (N.S.) 30 (1976), 3, 219-224.

[19] Z. I. Szabó, Structure theorems on Riemannian spaces satisfying $R(X, Y) \cdot R=0$. I. The local version, J. Differential Geometry 17 (1982), 4, 531-582. 